\title{
Surgical implications of variations in hand collateral circulation: Anatomy revisited
}

Permyos Ruengsakulrach, FRCST ${ }^{\mathrm{a}}$

Norman Eizenberg, MB, BS

Claude Fahrer, MB, BS ${ }^{b}$

Marius Fahrer, FRACS ${ }^{b}$

Brian F. Buxton, FRACS ${ }^{a}$
From the Department of Cardiac Surgery, Austin \& Repatriation Medical Centre, and the Department of Anatomy and Cell Biology, ${ }^{\mathrm{b}}$ The University of Melbourne, Melbourne, Victoria, Australia.

Received for publication Feb 2, 2001; revisions requested March 14, 2001; revisions received April 24, 2001; accepted for publication April 27, 2001

J Thorac Cardiovasc Surg 2001;122:682-6

Copyright (c) 2001 by The American Association for Thoracic Surgery

$0022-5223 / 2001 \$ 35.00+0 \quad \mathbf{1 2 / 1 / 1 1 6 9 5 1}$

doi:10.1067/mtc.2001.116951
Objectives: One of the risks associated with harvesting the radial artery is hand ischemia. Accordingly, this study investigated the variations of the hand collateral circulation.

Methods: Fifty hands of cadavers were examined. Variations of the palmar arches were recorded. A classic superficial palmar arch was defined as direct continuity between the ulnar artery and the superficial palmar branch of the radial artery. A classic complete deep palmar arch was defined as direct continuity between the radial artery and the deep branch of the ulnar artery.

Results: A classic superficial palmar arch was found in 10\% (5/50) of hands, and a classic complete deep palmar arch was found in 90\% (45/50) of hands. The superficial palmar branch of the ulnar artery supplied blood to all fingers in 66\% (33/50) of hands. Although the superficial palmar branch of the ulnar artery was continuous with the radial artery in only $34 \%$ (17/50) of hands (including the classic type of superficial palmar arch), every hand had at least one major branch connecting the radial and ulnar arteries.

Conclusions: Variations in the terminations of the radial and ulnar arteries are common. Although the classic type of superficial palmar arch occurs relatively infrequently, there is always a significant anastomosis between the radial and the ulnar artery in the hand. This anatomic study confirms the presence of a collateral supply in the hand. In the absence of vascular disease, harvesting the radial artery should be regarded as a safe procedure.

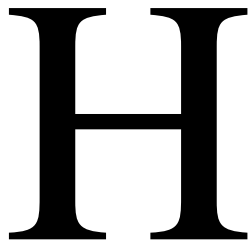

arvesting radial arteries (RAs) for use as arterial bypass conduits is an invasive procedure with potential risks, as suggested by sporadic reports of hand ischemia after RA removal. ${ }^{1}$ If we are to fully assess the feasibility of the RA as a bypass graft, an examination of the possible risks associated with harvesting the artery is needed.

The RA and ulnar artery (UA) provide most of the blood supply of the hand. Additional circulation may come from the median artery or the interosseous arterial system. The RA and UA form 4 circuits in the hand, the anterior and posterior carpal arch at the level of the carpal bones and the superficial and deep palmar arch at the midpalmar level. These arches form 3 circuits on the palmar side and 1 circuit on the dorsal side of the hand. The superficial and deep palmar arches are the most important of the circuits because they provide the blood supply to all of the fingers. 

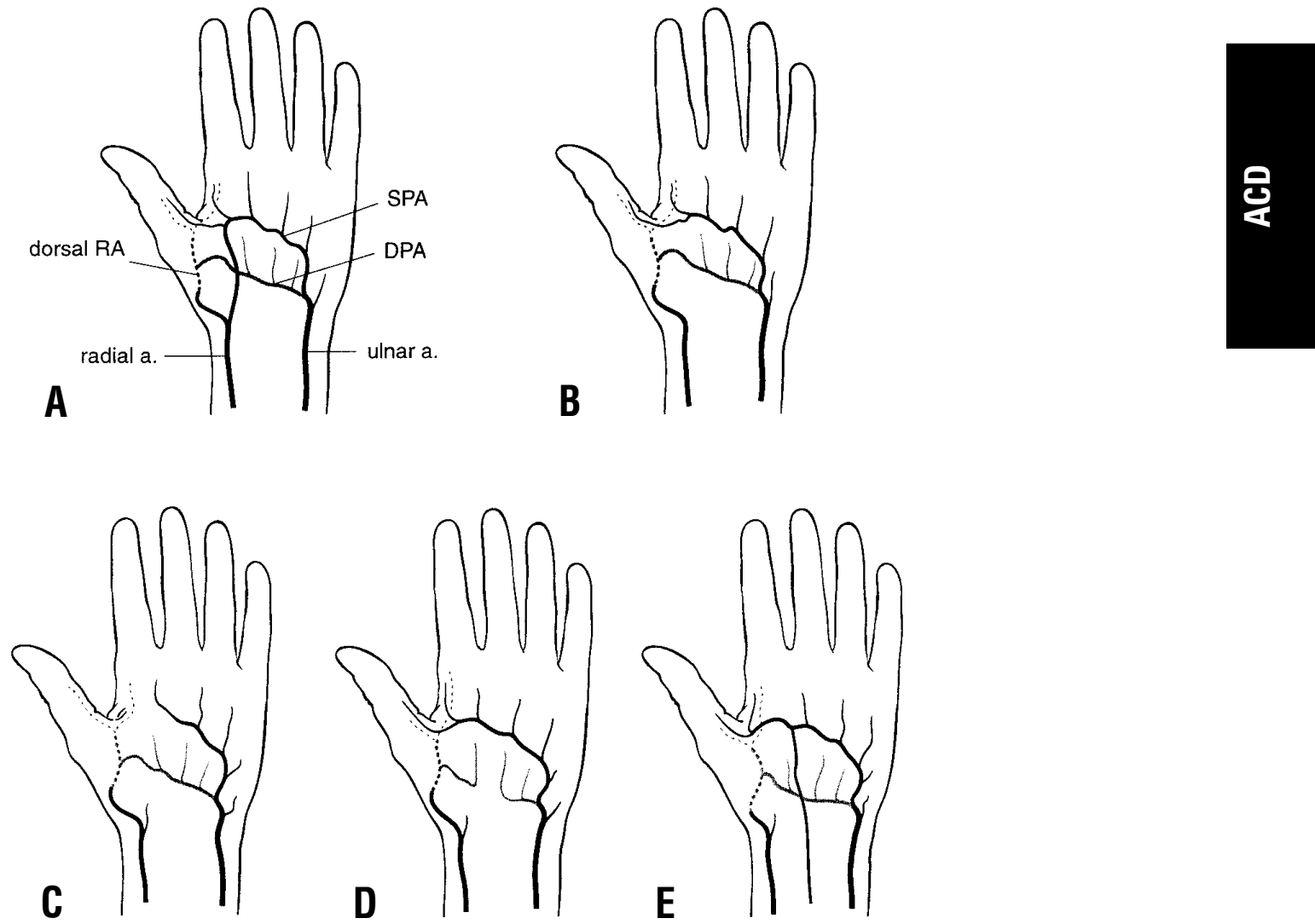

Figure 1. Variations of the hand collateral circulation, palmar aspect of left hand. The black line represents the superficial palmar arch, the gray line represents the deep palmar arch, and the dotted line represents the dorsal artery. A, Classic (and complete) superficial palmar arch: the superficial palmar arch from the UA supplies the index finger and thumb and anastomoses with the superficial palmar branch of the RA. B, Complete superficial palmar arch: the superficial palmar arch from the UA supplies the thumb. Complete deep palmar arch: the distal end of the deep palmar arch anastomoses with the deep palmar branch of the UA. C, Incomplete superficial palmar arch: the superficial palmar arch does not provide a metacarpal branch to supply the thumb. D, Incomplete deep palmar arch: no continuity is found between the deep palmar branch of the UA and the RA. E, Median artery. SPA, Superficial palmar arch; DPA, deep palmar arch.

Standard anatomy textbooks lack information about crucial variations in the arterial supply of the hand. Several studies have described the variations of the complex anatomy of the hand and forearm. However, these studies are limited by the fact that none of them look specifically for variations of the collateral circulation that might affect the decision to remove the RA, such as the presence of both an incomplete superficial palmar arch and an incomplete deep palmar arch in the same hand. Such information is very important when considering RA harvesting.

Accordingly, the aim of this study was to provide a comprehensive assessment of the anatomic variations of the arterial anastomoses in the hand, focusing in particular on the arterial connections between the superficial and deep palmar arches.

\section{Methods}

This study was carried out on 50 randomly selected hands from cadavers in the dissecting room of the Department of Anatomy and Cell Biology at the University of Melbourne. All of the cadavers had been embalmed soon after death. The dissection focused on the hands and sought to identify the communications between the RA and the UA. Sketches and photographs were used to document the dissected specimens.

\section{Results}

\section{Radial Artery}

The terminal branches of the RA at the wrist were the superficial palmar branch of the RA and the dorsal RA. The superficial palmar branch of the RA connected with the superficial palmar branch of the UA to form the superficial 

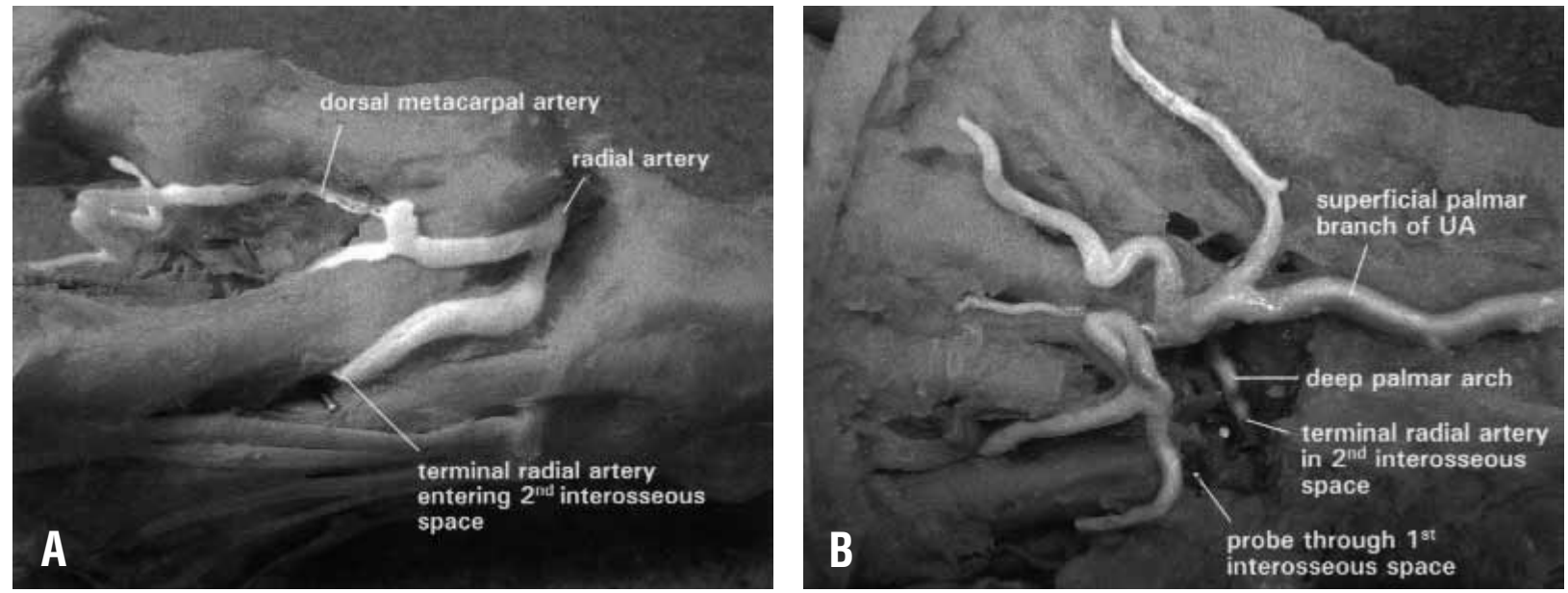

Figure 2. The origin of the deep palmar arch in which the RA passes to the palmar side through the second interosseous space. A, Dorsolateral aspect of the left wrist showing the origin of the deep palmar arch. B, Palmar aspect of the left wrist and hand.

TABLE 1. Summary of prevalence of incomplete and complete superficial and deep palmar arches

\begin{tabular}{lcccc}
\hline & \multicolumn{2}{c}{ Superficial palmar arch } & \multicolumn{2}{c}{ Deep palmar arch } \\
\cline { 2 - 4 } & Incomplete & Complete & Incomplete & Complete \\
\hline Coleman and Anson, 19612 & $21.5 \%$ & $78.5 \%$ & $3 \%$ & $97 \%$ \\
Ikeda et al, 19886 & $3.6 \%$ & $96.4 \%$ & $23.1 \%$ & $76.9 \%$ \\
Mezzogiorno et al, 19947 & & & $33.3 \%$ & $66.7 \%$ \\
Ruengsakulrach et al, 2001 & $34 \%$ & $66 \%$ & $10 \%$ & $90 \%$ \\
\hline
\end{tabular}

palmar arch in $10 \%(5 / 50)$ of hands (Figure 1, A). In the remaining $90 \%$ of cases, the superficial palmar branch of the RA was absent or small (Figure 1, B-E) and only supplied a minor part of the thenar muscles. The main RA passed to the dorsal side of the hand under the abductor pollicis longus and extensor pollicis brevis tendons. It then pierced the first interosseous space and formed the deep palmar arch with the deep palmar branch of the UA in $94 \%$ (47/50) of hands (Figure 1, $A-C$ and $E$ ). In 3 hands there were anatomic variations of the origin of the deep palmar arch. In these cases the RA formed the deep arch by passing through the second interosseous space (Figure 2, $A$ and $B$ ).

\section{Deep Palmar Arch}

Two types of deep palmar arches were found.

Complete deep palmar arch. In this variation the terminal portion of the deep palmar branch of the RA had a connection with the deep palmar branch of the UA. This type of arch was found in 90\% (45/50) of hands (Figure 1, $A-C$ and $E$ ).

Incomplete deep palmar arch. In this variation the deep palmar branch of the RA did not have any connection with the UA at the deep palmar level. This was found in $10 \%$ $(5 / 50)$ of hands (Figure $1, D)$.

\section{Ulnar Artery}

In all of the hands, the UA terminated by dividing into superficial palmar and deep palmar branches. The UA made no visible contribution to the dorsal side of the hand at the level of the wrist.

\section{Superficial Palmar Arch}

Two types of superficial palmar arch were found.

Complete superficial palmar arch. The superficial palmar arch was defined as complete when it supplied all the fingers and the ulnar side of the thumb. This variation was found in 66\% (33/50) of hands (Figure 1, A, B,D, and $E$ ).

Incomplete superficial palmar arch. The arch was defined as incomplete when it only supplied the little, ring, and middle fingers. This variation occurred in 34\% (17/50) of hands (Figure 1,C). 


\section{Superficial Palmar Branch of the UA}

The distal end of the superficial palmar arch of the UA communicated with the RA in only 34\% (17/50) of hands. There were 4 types of this communication between the superficial palmar branch of the UA and the RA at the level of the arches (Figure 3): (1) through the superficial palmar branch of the RA (the classic superficial palmar arch) in $10 \%$ (Figure 3, A); (2) through the digital branch of the dorsal RA in $18 \%$ (Figure $3, B$ ); (3) through the deep palmar arch of the RA in $4 \%$ (Figure 3,C); and (4) through the median artery and digital branch of the dorsal RA in 2\% (Figure 3, D).

\section{Median Artery}

A median artery was found in $3(6 \%)$ cadavers. One terminated at the wrist, and one anastomosed with the superficial palmar arch (Figure 1,E). The third median artery terminated by giving off branches to the index finger and thumb at the palmar side without anastomosing with the RA or UA.

\section{Combined Contributions of the UA and RA}

Even though the superficial palmar branch of the UA did not supply the thumb in about $34 \%$ of hands, a deep palmar branch of the UA anastomosed with the deep palmar branch of the RA in all cases. Similarly, even though an incomplete deep palmar arch was found in 10\% of hands, all of these cases had a complete superficial palmar arch.

\section{Discussion}

Our study found that the collateral blood supply of the hand is complex and often marked by variations. A number of variations of the superficial palmar arch of the UA were detected, with the most common type found in this study being the complete superficial palmar arch (66\%) and the complete deep palmar arch (90\%). As noted, even though the superficial palmar branch of the UA did not supply the thumb in about $34 \%$ of hands, a complete deep palmar arch was present in all of these hands, thereby enabling the RA to be safely harvested.

Our study confirmed the findings from a larger series reported by Coleman and Anson ${ }^{2}$ in 1961. Of 650 hands examined, they found a complete superficial palmar arch in $78.5 \%$ of hands. In this series a complete superficial palmar arch was recorded when the superficial palmar branches of the RA and UA anastomosed or when the UA reached the thumb and index finger. The classic type of superficial palmar arch (as typically demonstrated in anatomy textbooks), ${ }^{3-5}$ in which the superficial branch of the RA joins the superficial palmar arch of the UA, was found in $34.5 \%$ of hands. In our series this classic type of arch occurred more rarely, being found in only $10 \%$ of hands.

In contrast, in 1988, Ikeda and associates ${ }^{6}$ reported a high prevalence of complete superficial palmar arches using the same definition (96.4\% of 220 hands from cadavers).
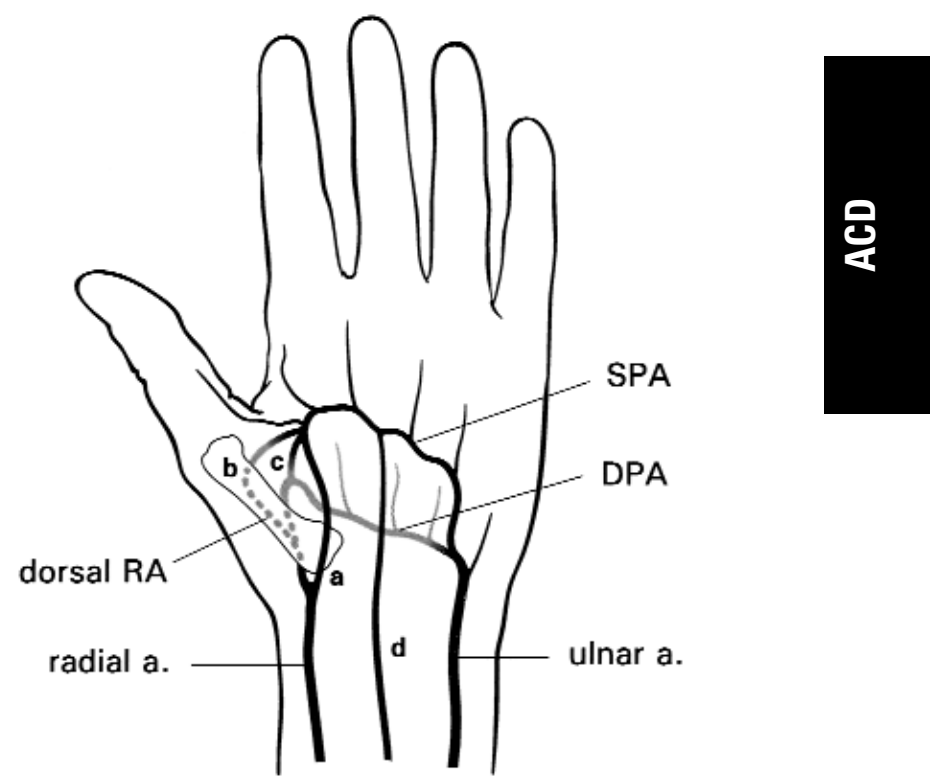

Figure 3. Four types of communication between the superficial palmar arch of the UA and the RA in the hand: $a$, through superficial palmar branch of RA (10\%); $b$, through deep palmar arch $(4 \%)$; $c$, through digital branch of the dorsal RA (18\%); and $d$, through median artery and digital branch of the dorsal RA (2\%). SPA, Superficial palmar arch; DPA, deep palmar arch.

They also found a higher proportion of classic superficial palmar arches (55.9\%). However, in this classic type the contribution of the RA was small in $34.6 \%$ of hands and was equal in size in only $21.3 \%$ of hands.

In our study we found the deep palmar arch to be variable in size. The size of the superficial palmar arch was inversely proportional to that of the deep palmar arch. Coleman and Anson $^{2}$ found a complete deep palmar arch in which the deep branch of the RA anastomosed with the deep branch of the UA in $97 \%$ of hands. Ikeda and associates ${ }^{6}$ found a complete deep palmar arch in $76.9 \%$ of hands. In our series we found this type of arch in $90 \%$ of hands. In contrast, in 1994, Mezzogiorno and colleagues ${ }^{7}$ found that the deep palmar arch was formed by the RA and UA in only $66.7 \%$ of 60 hands. In $21.7 \%$ of hands, the deep palmar arch was formed by the RA, and an anastomotic branch formed a digital artery. The deep palmar arch was formed solely by the RA or the UA in $8.3 \%$ and $3.3 \%$ of cases, respectively.

In the case of the incomplete deep palmar arch (found in $10 \%$ of the hands in our study), all these hands had complete superficial palmar arches. This implies that the thumb received its blood supply from the UA at the ulnar side and that the RA could be removed without endangering the circulation of the hand. Table 1 summarizes the prevalence of incomplete and complete superficial and deep palmar arches.

In 1978, Parks and colleagues ${ }^{8}$ published a study of the variations of the arterial supply of the thumb in 50 hands 
from cadavers. They found that, in $80 \%$ of hands, the arterial supply of the thumb usually came from the first palmar metacarpal artery (the first branch of the deep palmar arch of the RA). In 5\% of hands, a branch of the second palmar metacarpal artery formed the main artery of the thumb. The first dorsal metacarpal artery, which is a branch of the RA at the dorsal side, was the main artery to the thumb in $15 \%$ of hands. This latter result was consistent with our finding that $18 \%$ of hands had a digital branch of the dorsal RA, which supplied the thumb and anastomosed with the superficial palmar arch.

In 1999, Olave and Prates ${ }^{9}$ reported the findings of an anatomic study of both hands in 30 cadavers. In $13.3 \%$ of the hands, the deep palmar arch was formed by the RA passing through the second interosseous space, a variation we found in $6 \%$ of cases in our series. However, the significance of this variation in RA harvesting is minimal because the RA and UA both contribute blood supply to the arch.

Our study is unique in that the anastomoses between the RA and UA have been documented and correlated in individual hands. The major finding from the series of 50 hands examined was that there were no cases of an incomplete superficial and deep palmar arch being present in the same hand. From an anatomic perspective, this study confirms that it is safe to remove the RA from its origin proximally to the level of the wrist distally. However, in patients with coronary artery disease, vascular disease affecting the upper extremities may be present. In 1999, Rauch and colleagues ${ }^{10}$ reviewed 66 upper extremities from 60 patients who underwent angiography before skin-muscle transplantation (63.6\% of hands), creation of a hemodialysis fistula $(6.1 \%)$, or evaluation of vascular diseases $(30.3 \%)$. Classic superficial palmar arches and deep palmar arches were detected in $31.8 \%$ and $84.8 \%$ of the patients, respectively. These findings suggest that patients with coronary artery disease should be screened before RA harvesting to confirm the presence of a viable collateral circulation. Currently, the methods of assessing hand circulation include the modified Allen test, Doppler ultrasonography, photoplethysmography, and oximetric techniques. ${ }^{11-14}$ The modified Allen test is easily performed by the bedside and requires no special equipment; we use this technique as a primary screening test and use the Doppler ultrasound examination when the modified Allen test is abnormal or inconclusive. ${ }^{15}$

Although our results suggest the overall safety of RA harvesting, it must be noted that our study suffers from 2 limitations. First, we studied the anastomoses between the RA and UA only at the level of the palmar arches. Therefore, although we may have designated an arch as incomplete, it may have possessed anastomotic connections at the level of the digital circulation. Second, even though there is always an anatomic connection between the 2 arterial systems, such as that between the deep palmar arch of the RA and the superficial palmar arch of the UA, it is possible that these anastomoses may be insufficient. Thus, despite the presence of an anatomic collateral circulation, the blood supply may still be compromised when an RA is removed.

Our study indicates that anastomoses between the superficial and the deep palmar arch are complex and varied. However, in all of the hands we dissected, there was some degree of collateral circulation between the RA and the UA. This suggests that, at least from an anatomic perspective, it is safe to sacrifice one of these arteries. Such arterial interventions include RA cannulation, radial forearm flap, and particularly RA or UA harvesting for coronary artery bypass grafting. We still recommend testing the hand (by using the modified Allen test, Doppler ultrasonography, photoplethysmography, or oximetric techniques) before performing an arterial intervention to identify the occasional case in which collateral circulation is compromised by the presence of arterial disease.

We thank Dr Tania Lewis for her editorial assistance and Ms Elizabeth Croce for her excellent illustrations.

\section{References}

1. Nunoo-Mensah J. An unexpected complication after harvesting of the radial artery for coronary artery bypass grafting. Ann Thorac Surg. 1998;66:929-31.

2. Coleman SS, Anson BJ. Arterial patterns in the hand based upon a study of 650 specimens. Surg Gynecol Obstet. 1961;113:409-24.

3. Williams PL, Bannister LH, Berry MM, et al, editors. Gray's anatomy. 38th ed. New York: Churchill Livingstone; 1995.

4. Agur AM, Lee MJ. Grant's atlas of anatomy. 10th ed. Philadelphia: Lippincott, Williams \& Wilkins; 1999.

5. Moore KL, Dalley AF. Clinically oriented anatomy. 4th ed. Philadelphia: Lippincott, Williams \& Wilkins; 1999.

6. Ikeda A, Ugawa A, Kazihara Y, Hamada N. Arterial patterns in the hand based on a three-dimensional analysis of 220 cadaver hands. $J$ Hand Surg. 1988;13:501-9.

7. Mezzogiorno A, Passiatore C, Mezzogiorno V. Anatomic variations of the deep palmar arteries in man. Acta Anat. 1994;149:221-4.

8. Parks BJ, Arbelaez J, Horner RL. Medical and surgical importance of the arterial blood supply of the thumb. J Hand Surg. 1978;3:383-5.

9. Olave E, Prates JC. Deep palmar arch patterns in Brazilian individuals. Surg Radiol Anat. 1999;21:267-71.

10. Rauch D, Fischer C, Achenbach S, Klose KJ, Wagner HJ. Angiography detection of closed palmar arcs. Rofo Fortschr Geb Rontgenstr Neuen Bildgeb Verfahr. 1999;171:207-10.

11. Pola P, Serricchio M, Flore R, Manasse E, Favuzzi A, Possati GF. Safe removal of the radial artery for myocardial revascularization: a Doppler study to prevent ischemic complications to the hand. J Thorac Cardiovasc Surg. 1996;112:737-44.

12. Kochi K, Sueda T, Orihashi K, Matsuura Y. New noninvasive test alternative to Allen's test: snuff-box technique. J Thorac Cardiovasc Surg. 1999;118:756-8.

13. Johnson WH 3rd, Cromartie RS 3rd, Arrants JE, Wuamett JD, Holt JB Simplified method for candidate selection for radial artery harvesting. Ann Thorac Surg. 1998;65:1167.

14. Starnes SL, Wolk SW, Lampman RM, Shanley CJ, Prager RL, Kong $\mathrm{BK}$, et al. Noninvasive evaluation of hand circulation before radia artery harvest for coronary artery bypass grafting. J Thorac Cardiovasc Surg. 1999;117:261-6.

15. Ruengsakulrach P, Brooks M, Hare DL, Gordon I, Buxton B Preoperative assessment of hand circulation by means of Doppler ultrasonography and the modified Allen test. J Thorac Cardiovasc Surg. 2001;121:526-31. 